\title{
Oxidative Stability of Sunflower Oil Bodies
}

Ian D. Fisk, Daniel A. White, David A. Gray*

Division of Food Sciences, University of Nottingham, Sutton Bonington Campus, Loughborough LE12

5RD, U.K.

David.Gray@nottingham.ac.uk 


\section{$1 \quad$ ABSTRACT}

2 This study investigates the oxidative stability of sunflower oil body suspensions (10\% w/w lipid). Two

3 washed suspensions of oil bodies were evaluated over 8 days at three temperatures $\left(5^{\circ} \mathrm{C}, 25^{\circ} \mathrm{C}\right.$ and

$4 \quad 45^{\circ} \mathrm{C}$ ) against three comparable sunflower oil emulsions stabilized with dodecyltrimethylammonium

5 bromide (DTAB), polyoxyethylene-sorbitan monolaurate (tween 20) and sodium dodecyl sulphate

6 (SDS) $(17 \mathrm{mM})$. The development of oxidation was monitored by the production of lipid hydroperoxides

7 and hexanal. Oil body suspensions were significantly more stable to oxidation than the three emulsions

8 at each temperature. The reasons and biological demands for oxidative stability in-vivo are clear

9 although they have never been previously demonstrated ex-vivo.

10 Hexanal, lipid hydroperoxides, oil body, oxidation, sunflower, surfactants. 
13 Oxidation of lipids in food can result in the production of anti-nutritional factors and undesirable

14 flavour and aroma compounds, the process of oxidation may also generate genotoxic and cytotoxic 15 oxygenated aldehydes (1). Knowledge and control of lipid oxidation is therefore fundamental to the 16 preservation of a food's nutritional and sensory quality, and the ability to limit or delay the onset of oxidation is important to the food industry.

18 Tocopherol (vitamin E) can confer increased oxidative stability to lipid emulsions through an 19 extended induction period due to scavenging of lipid peroxyl radicals (2). Recent work has shown that 20 seed tocopherol is an intrinsic component of both oilseed and cereal grain oil bodies (3-5). Oil bodies 21 are discrete plant organelles found principally within desiccation resistant oilseeds; their function is to store neutral lipids during seed dormancy. They are formed of a neutral lipid core surrounded by a 23 phospholipid monolayer, associated phytochemicals and a coat of strongly amphiphilic proteins $(6,7)$.

24 Oil bodies therefore offer oxidative protection to seed oil in-vivo. It is not known however, if oil bodies recovered from oilseeds are stable against oxidation in-vitro.

In formulated emulsions, surfactant chemistry has the potential to significantly effect the development of oxidation through the promotion of metal catalyzed oxidation $(8)$ or the presence of residual surfactant hydroperoxides (9). A study by Mancuso et al. (8) used a range of surfactants at equimolar concentrations of cationic, anionic and non-ionic surface charge to illustrate their potential to effect oxidative stability.

31 Oil bodies from sunflower (Helianthus annus) were extracted and washed at two purification levels 32 (water washed and salt washed) to remove extraneous proteins (4); these natural suspensions were then assessed for oxidative stability at different temperatures in comparison to sunflower oil emulsions stabilized with a range of synthetic surfactants (cationic dodecyltrimethylammonium bromide (DTAB), nonionic tween 20 and anionic sodium dodecyl sulphate (SDS)) to validate their potential as natural food emulsions. 


\section{$39 \quad$ Materials}

40 All Chemicals are analytical grade or higher and were sourced from Fisher UK (Loughborough, UK)

41 unless otherwise stated. Surfactants were sourced from Sigma-Aldrich Co. ( St Lewis, MO, USA)

\section{$42 \quad$ Methods}

Oil Body Recovery. Water washed and salt washed oil bodies were prepared as described previously

(4). In brief, dehulled sunflower seeds were wet milled, the resulting slurry filtered and the filtrate centrifuged to isolate the buoyant oil body fraction. Oil bodies were water washed by resuspension in buffer (10mM sodium phosphate buffer, $\mathrm{pH} 7.5)$ and recentrifugation. This process was repeated in $1 \mathrm{M}$ sodium chloride buffered solution and again in buffer to yield salt washed oil bodies.

Lipid Extraction. Moisture content of oil body and seed samples (5g) was determined gravimetrically (10).

Emulsion Formulation. Oil body suspensions at 10\% (w/w) lipid were formulated with sodium azide as a preservative $(0.02 \mathrm{mM})$ and stored at $5^{\circ} \mathrm{C}$ under nitrogen prior to use. Emulsions were formulated extraction, excess solvent was removed by drying under nitrogen. Extracted lipid was added slowly to surfactant solutions (dodecyltrimethylammonium bromide, tween 20 and sodium dodecyl sulphate through an Emulsiflex-C5 (Glan Creston, Stanmore, UK) at an emulsification pressure of 10,000kPa. WERKE, Staufer, Germany) at 10,000 rpm, the emulsion was further homogenized by three passes

Particle Size Distribution. Mean droplet diameter was measured by use of a low angle light scattering detector (Malvern Mastersizer, Malvern Instruments, England) and represented as the numerical mean droplet diameter, $\mathrm{D}(\mathrm{v}, 0.5)$.

Protein Characterization. Isolated oil bodies $(0.1 \mathrm{~g})$ were suspended in distilled water $(1 \mathrm{~mL})$ and vortexed (2min); to which $100 \mu \mathrm{L}$ of $100 \%$ TCA (w/v) was added. The tube was chilled on ice for 30 
min to promote the precipitation of proteins and centrifuged $(13,000 \mathrm{rpm}$ for $5 \mathrm{~min})$. The supernatant was aspirated and discarded and the pellet vortexed with $200 \mu \mathrm{L}$ SDS solution $(10 \% \mathrm{w} / \mathrm{v}$ SDS, $10 \mathrm{mM} \beta$ mercapto-ethanol, $20 \% \mathrm{v} / \mathrm{v}$ glycerol, $0.2 \mathrm{M}$ tris- $\mathrm{HCl}, \mathrm{pH} 6.8,0.05 \%$ bromophenol blue.) Proteins were resolved by SDS-PAGE using $15 \%$ and $4.0 \%$ polyacrylamide gels in the separating and stacking gel respectively. After electrophoresis the gel was stained with BioRad coomassie blue (R-250) and destained with excess methanol:water:acetic acid (9:9:2 v/v).

Zeta Potential. Zeta potential measurements were carried out using a Malvern Zetasizer IV (Malvern instruments, UK) $(11,12)$. Oil bodies $(20 \mathrm{mg}$ dry weight basis) were suspended in $10 \mathrm{mM}$ sodium phosphate buffer, $\mathrm{pH} 7.5$ (10ml, $0.2 \mu \mathrm{m}$ filtered) and sonicated $(10 \mathrm{~min})$. The suspension was then further diluted to achieve a final concentration of $30 \mu \mathrm{g} \cdot \mathrm{mL}^{-1}$. Suspension buffer was adjusted prior to the addition of sample to $\mathrm{pH}$ values ranging between $\mathrm{pH} 2.0$ and $\mathrm{pH} 12.0$.

Lipid Hydroperoxides. Lipid hydroperoxide concentration was measured using the method of Shantha and Decker (13) as modified by Nuchi et al.(9).

Hexanal Headspace Concentration. Hexanal concentration was calculated from the headspace concentration using solid phase micro-extraction gas chromatography mass spectroscopy (SPMEGCMS) (14). This was achieved by adding 1,2 dichlorobenzene (100ppmv) as an internal standard $(10 \mu \mathrm{l})$ to the emulsion or oil body suspension $(1 \mathrm{~mL})$ and capping in a $20 \mathrm{~mL}$ vial. Headspace concentration was determined using a CTS Analytics PAL system autosampler and a DSQ and Trace GC Ultra (Thermo Electron Corporation). Samples were agitated $\left(50^{\circ} \mathrm{C}\right.$ for $\left.3 \mathrm{~min}\right)$ prior to extraction $\left(20 \mathrm{~min}\right.$ at $\left.50^{\circ} \mathrm{C}\right)$, desorption was achieved in $5 \mathrm{~min}\left(250^{\circ} \mathrm{C}\right)$. Compounds were separated with 30mL.min ${ }^{-1}$ nitrogen, a ZB-5 Phenomenex gas chromatography column (Macclesfield, UK) and oven temperatures controlled at $40^{\circ} \mathrm{C}(1 \mathrm{~min})$ then ramped $\left(3^{\circ} \mathrm{C} \cdot \mathrm{min}^{-1}\right)$ to $140^{\circ} \mathrm{C}$, ramped $\left(15^{\circ} \mathrm{C} \cdot \mathrm{min}^{-1}\right)$ to $210^{\circ} \mathrm{C}$ and held at $210^{\circ} \mathrm{C}$ for $1 \mathrm{~min}$. Volatiles were quantified with authentic standards.

Oxidation Conditions. Samples were stored at $5{ }^{\circ} \mathrm{C}, 25^{\circ} \mathrm{C}$ and $45^{\circ} \mathrm{C}$ with restricted lighting in closed $20 \mathrm{~mL}$ glass vials.

Statistics. The experiment was carried out in a fully balanced, randomized experimental design using triplicate samples for each data point. Statistical analysis was performed by one-way ANOVA $(\mathrm{P}<0.05)$. 


\section{RESULTS AND DISCUSSION}

94 The development of oxidation in two oil body suspensions was assessed by comparison with oil-inwater emulsions stabilized by SDS, tween 20 and DTAB. Droplet size, lipid content and initial hydroperoxide concentration were measured and controlled to minimize inter-sample variation. The presence of hydroperoxides and production of hexanal were used as markers for the state of oxidation over the time course of the experiment. Oil bodies were prepared by wet-milling, centrifugation and subsequent water washing, this "water washed" preparation contained residual proteins not directly associated with oil bodies; the isolate was then salt washed to clean up the preparation and water washed again producing a "salt washed" preparation with fewer contaminating proteins. experimental time course and did not phase separate. DTAB stabilized emulsions did increase in droplet diameter slightly (21\% increase over 8 days) when exposed to elevated temperatures (Table 1). Measured oil body droplet diameter also increased; this was due to aggregation as light microscopy proved that although the oil bodies were clumping they were still intact entities (data not shown). Increased emulsion droplet size is theorized by McClements and Decker (15) to reduce oxidation rate, as the surface area on which oxidation chemistry can occur is reduced, a more recent paper by Osborn and Akah (16) found no significant effect of surface area on systems with low hydroperoxide concentrations. These results supported McClements' and Decker's (15) theory that droplet size is only

111 influential when reactant concentration (hydroperoxides) at the surface is high. In systems of a high 112 hydroperoxide concentration a doubling of the surface area would double the number of available 113 reactants, in a low concentration system this would not be true as all reactants would be available on the 114 droplet surface. In our experimental design we can make the assumption that droplet size will not 115 influence oxidation as samples have a low hydroperoxide concentration.

116 Hydroperoxide Formation. Oxidation did not occur in either of the oil body isolates at most 117 temperatures, although lipid hydroperoxide concentration did increase slightly in the $25^{\circ} \mathrm{C}$ salt washed 118 suspension and more significantly at $45^{\circ} \mathrm{C}$ in the water washed oil body suspension (Figure 1). The 119 concentration of hydroperoxides in all samples, apart from the $45^{\circ} \mathrm{C}$ water washed samples, are below 
the level usually associated with poor oil quality. Frankel (2) states a lipid hydroperoxide concentration

121 of 10 mequiv. $\mathrm{kg}^{-1}$ oil as the limit of acceptability for polyunsaturated vegetable oils, this would equate 122 to a value of $5.5 \mathrm{mmol}$ hydroperoxides. $\mathrm{kg}^{-1}$ oil.

123 Water washed oil bodies contain a number of residual proteins originating from the seed cell debris, 124 upon salt washing this number is selectively reduced, and only the more strongly associated proteins 125 remain (Figure 2). Protein oxidation is known to be concurrent with lipid oxidation in many foods and 126 is proposed to initially retard lipid oxidation by preferentially oxidizing over lipid species or through the 127 chelation of free metal ions $(17,18)$. Proteins may also initiate lipid oxidation through the formation of 128 peroxyl radicals or reactive carbonyls, this balance between pro-oxidant and antioxidant effects is both 129 concentration and matrix dependant (19). In the water washed oil body suspension at $45^{\circ} \mathrm{C}$ residual 130 contaminating proteins may have contributed to the increased hydroperoxide concentration relative to 131 the salt washed suspension through this pro-oxidant effect (Figure 1); although it should be noted that 132 the difference in concentration is minor when compared to the surfactant stabilized emulsions and it 133 could be hypothesized that the strongly associated proteins contribute an overall protection against 134 oxidation through free radical scavenging or the chelation of pro-oxidant metal ions.

135 The artificial emulsions were formulated from sunflower seed oil extracted from the same seed batch 136 as the oil bodies, and in all cases the starting lipid hydroperoxide concentrations were similar $(1.9 \pm 0.75$ 137 mmol hydroperoxides. $\mathrm{kg}^{-1}$ lipid). Figure 1 and Figure 3 allow a comparison of the oxidative stability of 138 oil bodies and the artificial emulsions. In all cases, except tween 20 at $25^{\circ} \mathrm{C}$, natural oil bodies were 139 significantly more stable against oxidation than the artificial emulsions made from the same oil base. 140 The extent of oxidation was dependant on the time and the nature of the emulsifier in the order DTAB $>$ 141 SDS $>$ tween 20 (based on the maximum hydroperoxide concentration over the experimental time 142 course) (Figure 3).

143 Development of oxidation was dependant upon temperature in the order $\left(45^{\circ} \mathrm{C}>25^{\circ} \mathrm{C}>5^{\circ} \mathrm{C}\right)$, samples 144 stored at higher temperatures oxidized more rapidly than those stored at $5^{\circ} \mathrm{C}$. Thermal dependence 145 concurs with classical models of oxidation and the concentration of hydroperoxides is in line with 146 literature values during early stages of oxidation (13). 
Secondary Product Formation. No increase in hexanal concentration could be detected in any of the oil body samples (Figure 4), indicating that hydroperoxide break down was minimal during the time course of the experiment. It is interesting to note that oil body samples did contain endogenous hexanal at the start of the experiment at relatively high levels; alternative volatile secondary products of linoleic acid oxidation were also followed but did not indicate oxidation (data not shown). The presence of endogenous hexanal has been noted previously in the hydrophobic subcompartments of carnation petals by Hudak and Thompson (20), who suggest that these structures resemble oil bodies and contain a number of volatile compounds including hexanal that originated as byproducts of the lipoxygenase pathway.

Hydroperoxides were formed in the surfactant stabilized emulsions increasing to levels associated with oil just prior to the development of oxidative rancidity. Hexanal, 2-Heptanal (data not shown) and 1-octen-3-one (data not shown) headspace data supported this and their concentrations increased in DTAB samples at all temperatures; this was also found in the SDS stabilized emulsions at $45^{\circ} \mathrm{C}$ (Figure

\section{3 and Figure 5).}

161 Three surfactants were chosen to test the effect that surfactant chemistry has on the development of oxidation. It would be expected that the most negatively charged emulsion (SDS) would oxidize more rapidly than the emulsion formed from the non-ionic surfactant (tween 20) as the SDS emulsion would attract cationic ions that may facilitate the initiation of lipid oxidation (2), this can be seen when we compare the SDS and the tween 20 stabilized emulsions (Figure 3). Surprisingly, hydroperoxides were formed most rapidly in emulsions stabilized by cationic DTAB. DTAB stabilized emulsions had the

167 highest hydroperoxide concentration at the start of the experiment (hydroperoxide concentration of 168 DTAB, SDS and tween 20 stabilized emulsions prior to storage were $2.96 \pm 0.31,2.66 \pm 0.18$ and $169 \quad 1.77 \pm 0.27 \mathrm{mmol}$ hydroperoxides. $\mathrm{kg}^{-1}$ lipid respectively). This may indicate DTAB naturally contained 170 hydroperoxides that could have promoted the development of oxidation resulting in the rapid production 171 of further hydroperoxides. Oil bodies are negatively charged (zeta potential of water washed and salt 172 washed oil bodies at $\mathrm{pH} 7.5$ are $-27 \mathrm{mV} \pm 1.4 \mathrm{mV}$ and $-30 \mathrm{mV} \pm 3.1 \mathrm{mV}$ respectively) but despite this 
173 potential to attract oxidation-promoting cations they are significantly more resistant to oxidation than 174 emulsions formulated from synthetic emulsifiers.

175 In conclusion, oil bodies are stable against oxidation at $5^{\circ} \mathrm{C}$, and when stored at elevated temperatures 176 hydroperoxide formation is significantly less than within emulsions formulated with synthetic 177 surfactants. The level of purity of oil bodies also effects oxidation rate, the more purified preparations 178 showing a further reduction in hydroperoxide formation. The oxidative stability of sunflower oil bodies 179 ex-vivo demonstrates protection of the naturally encapsulated polyunsaturated oil; oil bodies therefore 180 merit further study in specific industrial applications. 
183 (1) Guillen M, D.; N., C.; Ibargoitia M, L.; A., R. Study of both sunflower oil and its headspace

184

185

186

187

188

189

190

191

192

193

194

195

196

197

198

199

200

201

202

203

204

205

206

207

208

209

210

211

212

213

214

215

216

217

218

219

220

throughout the oxidation process. Occurrence in the headspace of toxic oxygenated aldehydes. $J$ Agric. Food Chem., 2005, 53, (4), 1093-1101.

(2) Frankel, E. N. Lipid Oxidation. The Oily Press: Dundee, 1998; Vol. 10.

(3) White, D. A.; Fisk, I. D.; Gray, D. A. Characterisation of oat (Avena sativa L.) oil bodies and intrinsically associated E-vitamers. J. Cereal Sci., 2006, 43, 244-249.

(4) Fisk, I. D.; White, D. A.; Carvalho, A.; Gray, D. A. Tocopherol - An intrinsic component of sunflower seed oil bodies. J. Am. Oil Chem. Soc., 2006, 83, (4), 341-344.

(5) Fisk, I. D.; White, D. A.; Carrier, L. M.; Gray, D. A. In Oil bodies and their associated phytochemicals Conference of Food Structure and Food Quality, Cork, Ireland, 3-7 October, 2004; AOCS: Cork, Ireland, 2004.

(6) Frandsen, G. I.; Mundy, J.; Tzen, J. T. C. Oil bodies and their associated proteins, oleosin and caleosin. Physiol. Plantarum, 2001, 112, (3), 301-307.

(7) Huang, A. H. C. Oil Bodies and oleosins in seeds. Ann. Rev. Plant Biol., 1992, 43, 177-200.

(8) Mancuso, J. R.; McClements, D. J.; Decker, E. A. The effects of surfactant type, pH, and chelators on the oxidation of salmon oil-in-water emulsions. J. Agr. Food Chem., 1999, 47, (10), $4112-4116$.

(9) Nuchi, C. D.; McClements, D. J.; Decker, E. A. Impact of tween 20 hydroperoxides and iron on the oxidation of methyl linoleate and salmon oil dispersions. J. Agr. Food Chem., 2001, 49, (10), $4912-4916$.

(10) Murphy, D. J.; Cummins, I. Seed oil-bodies - isolation, composition and role of oil-body apolipoproteins. Phytochemistry, 1989, 28, (8), 2063-2069.

(11) Illum, L.; Jacobsen, L. O.; Muller, R. H.; Mak, E.; Davis, S. S. Surface characteristics and the interaction of colloidal particles with mouse peritoneal-macrophages. Biomaterials, 1987, 8, (2), 113-117.

(12) Stolnik, S.; Davies, M. C.; Illum, L.; Davis, S. S.; Boustta, M.; Vert, M. The preparation of sub200nm biodegradable colloidal particles from poly(beta-malic acid-co-benzyl malate) copolymers and their surface modification with poloxamer and poloxamine surfactants. $J$. Control. Release, 1994, 30, (1), 57-67.

(13) Shantha, B. C.; Decker, E. A. Rapid, sensitive, iron-based spectrophotometric methods for determination of peroxide values of food lipids. J. AOCS Int., 1994, 77, (2), 421424.

(14) Beltran, G.; Aguilera, M. P.; Gordon, M. H. Solid phase microextraction of volatile oxidation compounds in oil-in-water emulsions. Food Chem., 2005, 92, (3), 401-406.

(15) McClements, D. J.; Decker, E. A. Lipid oxidation in oil-in-water emulsions: impact of molecular environment on chemical reactions in heterogeneous food systems. Concise Rev. Food. Sci., 2000, 65, (8), 1270-1282.

(16) Osborn, H. T.; Akoh, C. C. Effect of emulsifier type, droplet size, and oil concentration on lipid oxidation in structured lipid-based oil-in-water emulsions. Food Chem., 2004, 84, (3), 451-456. 
(17) Elias, R. J.; McClements, D. J.; Decker, E. A. Antioxidant activity of cysteine, tryptophan, and methionine residues in continuous phase beta-lactoglobulin in oil-in-water emulsions. J. Agric. Food. Chem., 2005, 53, 10248-10253.

(18) Faraji, H.; McClements, D. J.; Decker, E. A. Role of continuous phase protein on the oxidative stability of fish oil-in-water emulsions. J. Agr. Food Chem., 2004, 52, (14), 4558-4564.

(19) Villiere, A.; Viau, M.; Bronnec, I.; Moreau, N.; Genot, C. Oxidative stability of bovine serum albumin- and sodium caseinate-stabilized emulsions depends on metal availability. J. Agr. Food Chem., 2005, 53, (5), 1514-1520.

(20) Hudak, K. A.; Thompson, J. E. Subcellular localization of secondary lipid metabolites including fragrance volatiles in carnation petals. Plant Physiol., 1997, 114, (2), 705-713. 
Table 1: Variation in droplet diameter of sunflower oil bodies over 8 days stored at $5^{\circ} \mathrm{C}, 25^{\circ} \mathrm{C}$ and $45^{\circ} \mathrm{C}$. ${ }^{a}$ numerical mean diameter.

\begin{tabular}{|c|c|c|c|c|}
\hline \multirow[b]{3}{*}{ Water Washed } & \multicolumn{4}{|c|}{$\mathrm{D}(\mathrm{v}, \mathbf{0 . 5}) \mu \mathrm{m}$} \\
\hline & Day 0 & Day $8\left(5^{\circ} \mathrm{C}\right)$ & Day $8\left(25^{\circ} \mathrm{C}\right)$ & Day $8\left(45^{\circ} \mathrm{C}\right)$ \\
\hline & 0.91 & $4.95 \pm 1.75$ & $17.42 \pm 4.97$ & $9.72 \pm 2.00$ \\
\hline Salt Washed & 0.90 & $6.15 \pm 0.26$ & $9.72 \pm 2.00$ & $16.06 \pm 13.52$ \\
\hline SDS & 0.78 & $0.31 \pm 0.01$ & $0.32 \pm 0.01$ & $0.31 \pm 0.01$ \\
\hline Tween-20 & 0.32 & $0.33 \pm 0.01$ & $0.33 \pm 0.02$ & $0.32 \pm 0.01$ \\
\hline DTAB & 0.29 & $0.29 \pm 0.01$ & $0.32 \pm 0.02$ & $0.35 \pm 0.02$ \\
\hline
\end{tabular}



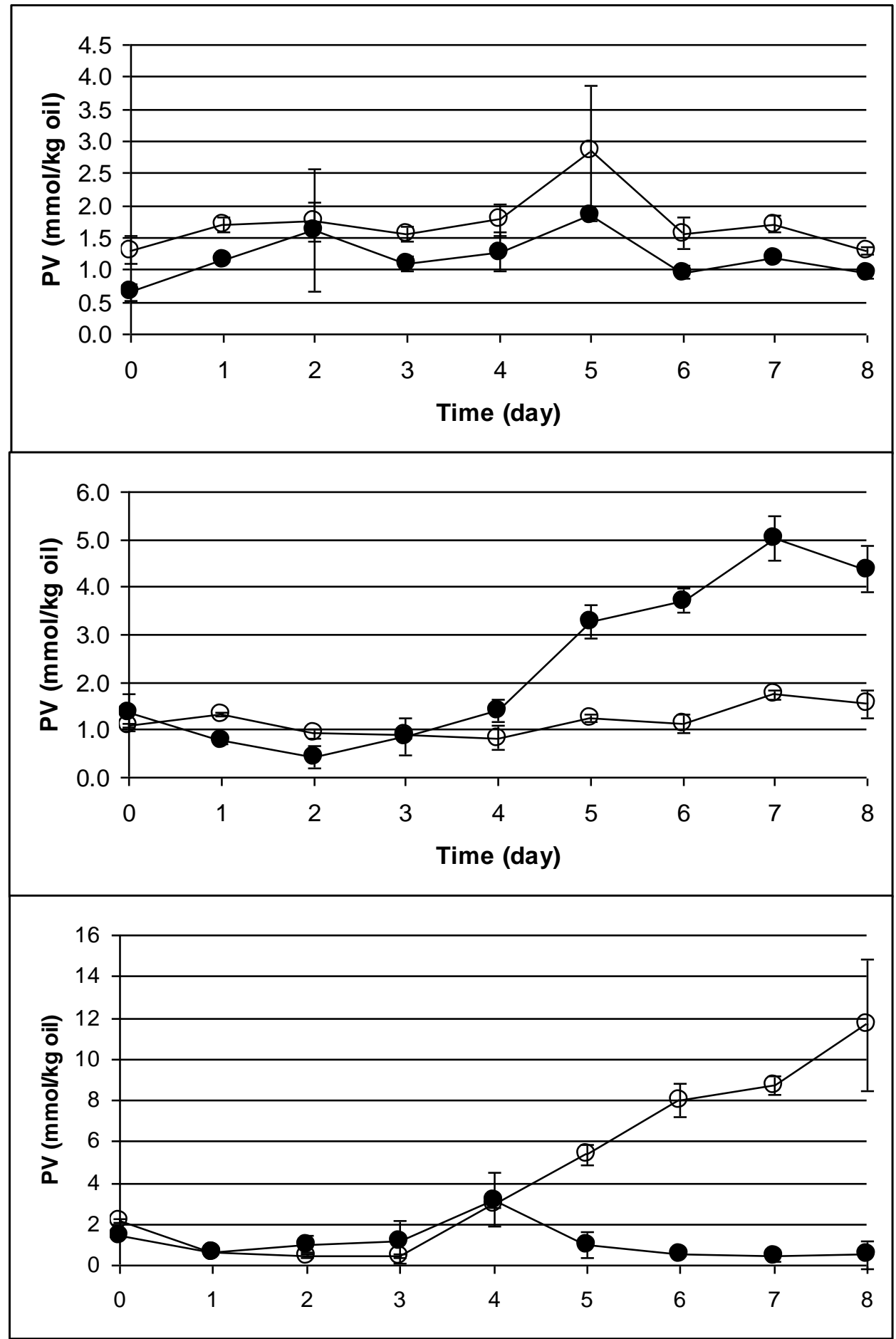

Figure 1: Peroxide Values mmol. $\mathrm{kg}^{-1}$ oil for water washed (empty circles) and salt washed oil bodies (filled circles). Samples were analysed at (a) $5^{\circ} \mathrm{C}$ (b) $25^{\circ} \mathrm{C}$ and (c) $45^{\circ} \mathrm{C}$. All data points represent means $(\mathrm{n}=3) \pm$ Standard Deviation. 


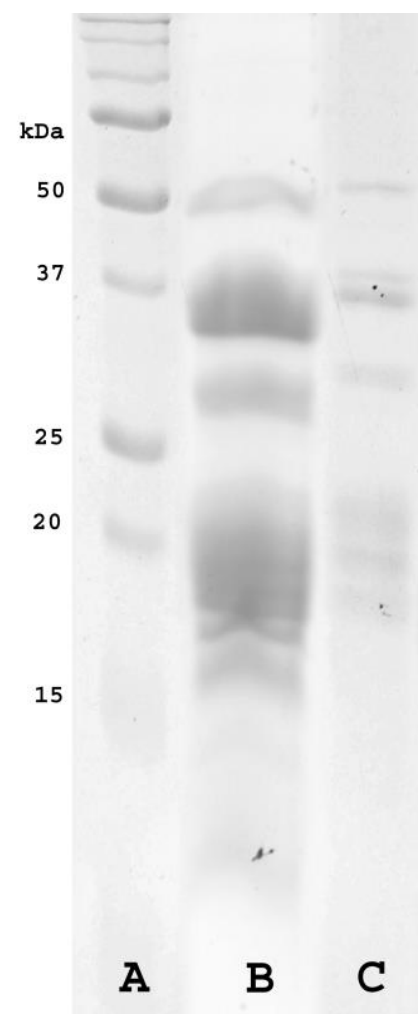

Figure 2: SDS-PAGE of proteins from sunflower oil bodies. A. Protein standards of Mw as indicated; B. Water washed oil body preparation; C. Salt washed oil body preparation. Lane B illustrates a number of proteins associated to water washed oil bodies, these residual proteins are selectively removed by salt washing. Which can be clearly observed in lane $\mathrm{C}$, in which a salt washed preparation is shown and the major remaining proteins are oleosin isoforms which range from $18 \mathrm{kDa}$ to $22 \mathrm{kDa}$. 


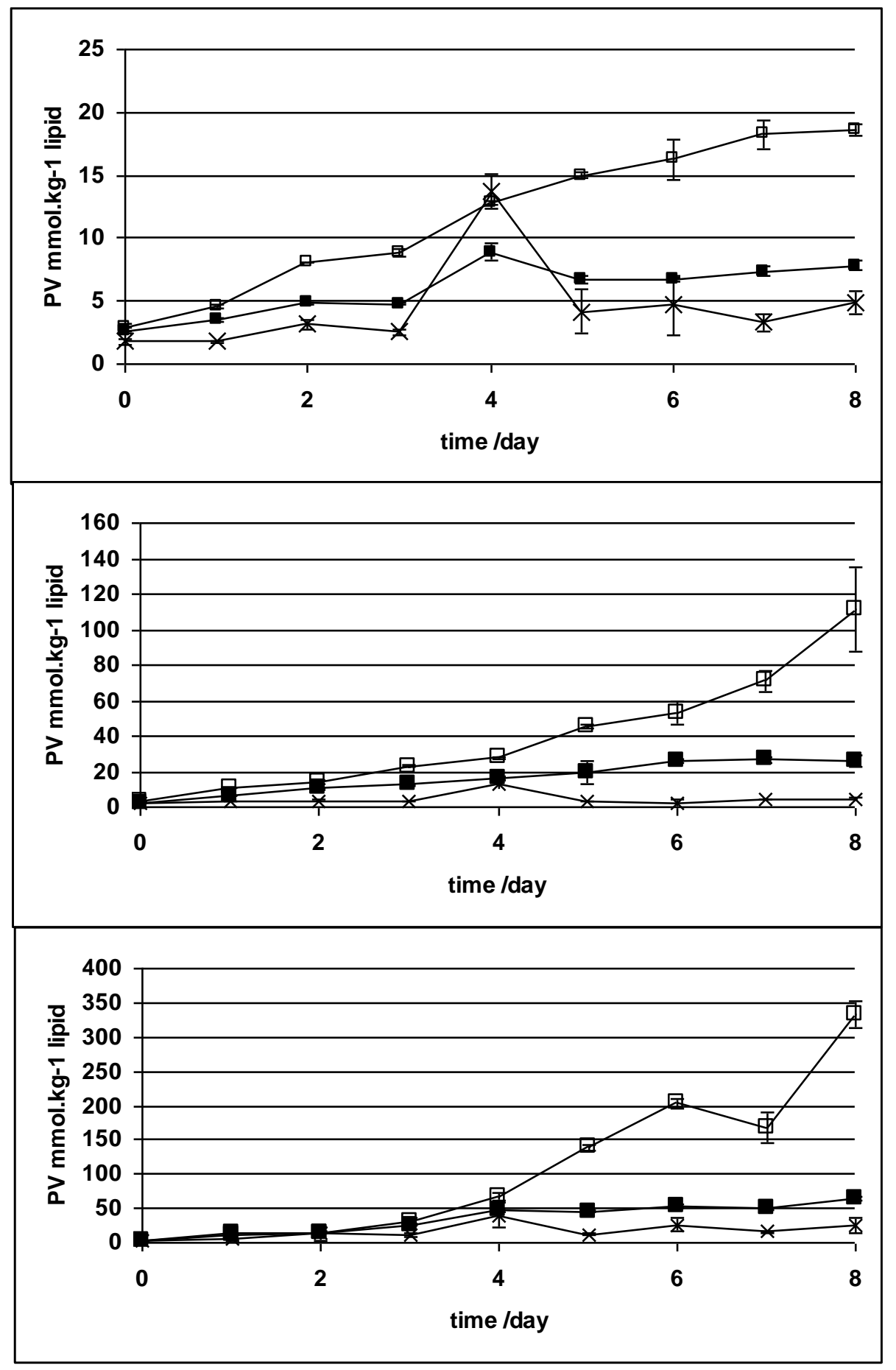

Figure 3: Peroxide Values mmol.kg ${ }^{-1}$ oil for $10 \%$ lipid emulsions stabilised by SDS (filled square), Tween 20 (cross) and DTAB (empty square). Extraction by solid phase microextraction and quantification by GC-MS. Emulsions were analysed at (a) $5^{\circ} \mathrm{C}$ (b) $25^{\circ} \mathrm{C}$ and (c) $45^{\circ} \mathrm{C}$. All data points represent means $(\mathrm{n}=3) \pm$ Standard Deviation 


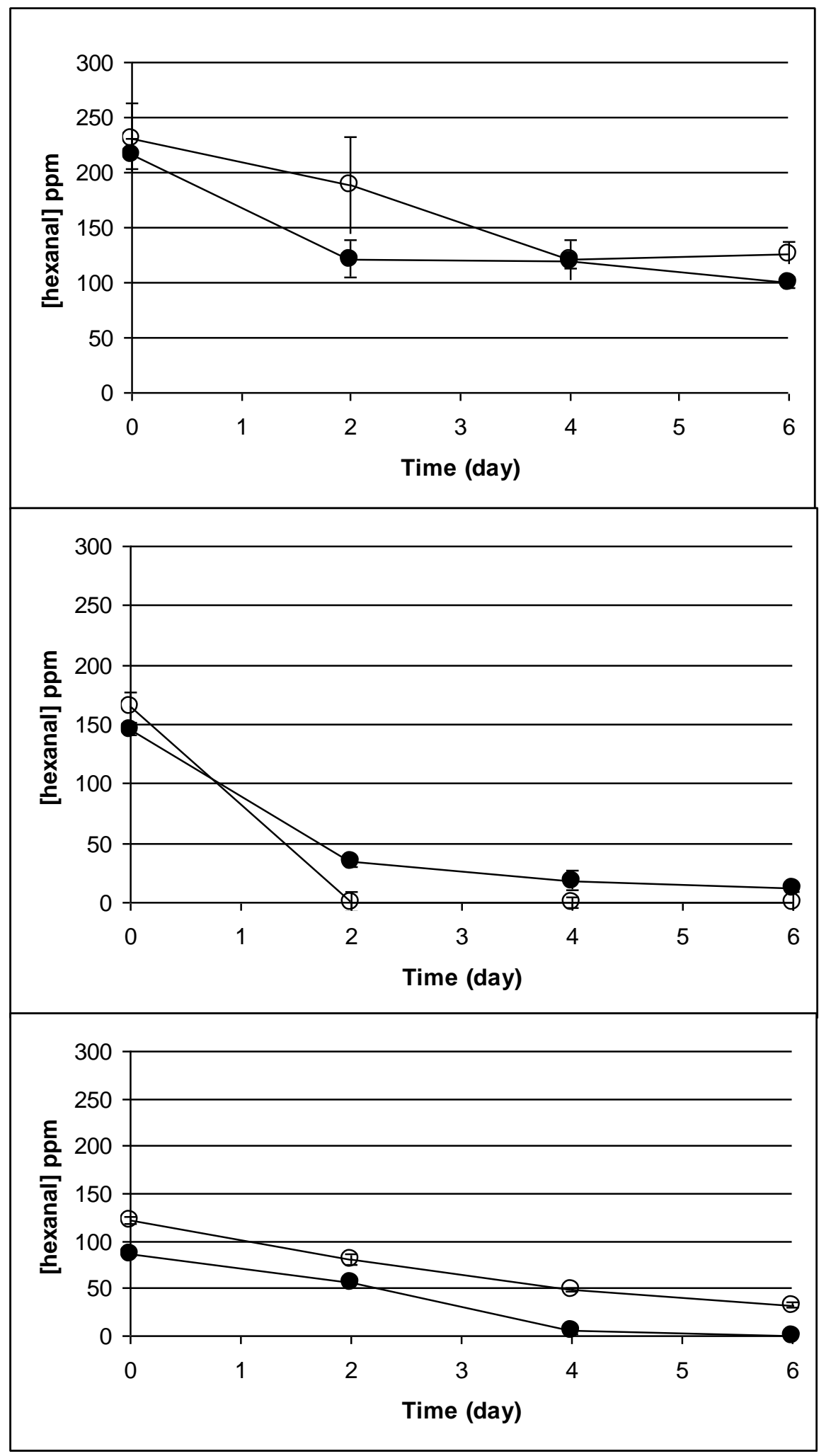

Figure 4: Influence of temperature on hexanal production in water washed (empty circles) and salt washed oil bodies (filled circles). Samples were analysed at (a) $5^{\circ} \mathrm{C}$ (b) $25^{\circ} \mathrm{C}$ and (c) $45^{\circ} \mathrm{C}$. All data points represent means $(\mathrm{n}=3) \pm$ Standard Deviation. 


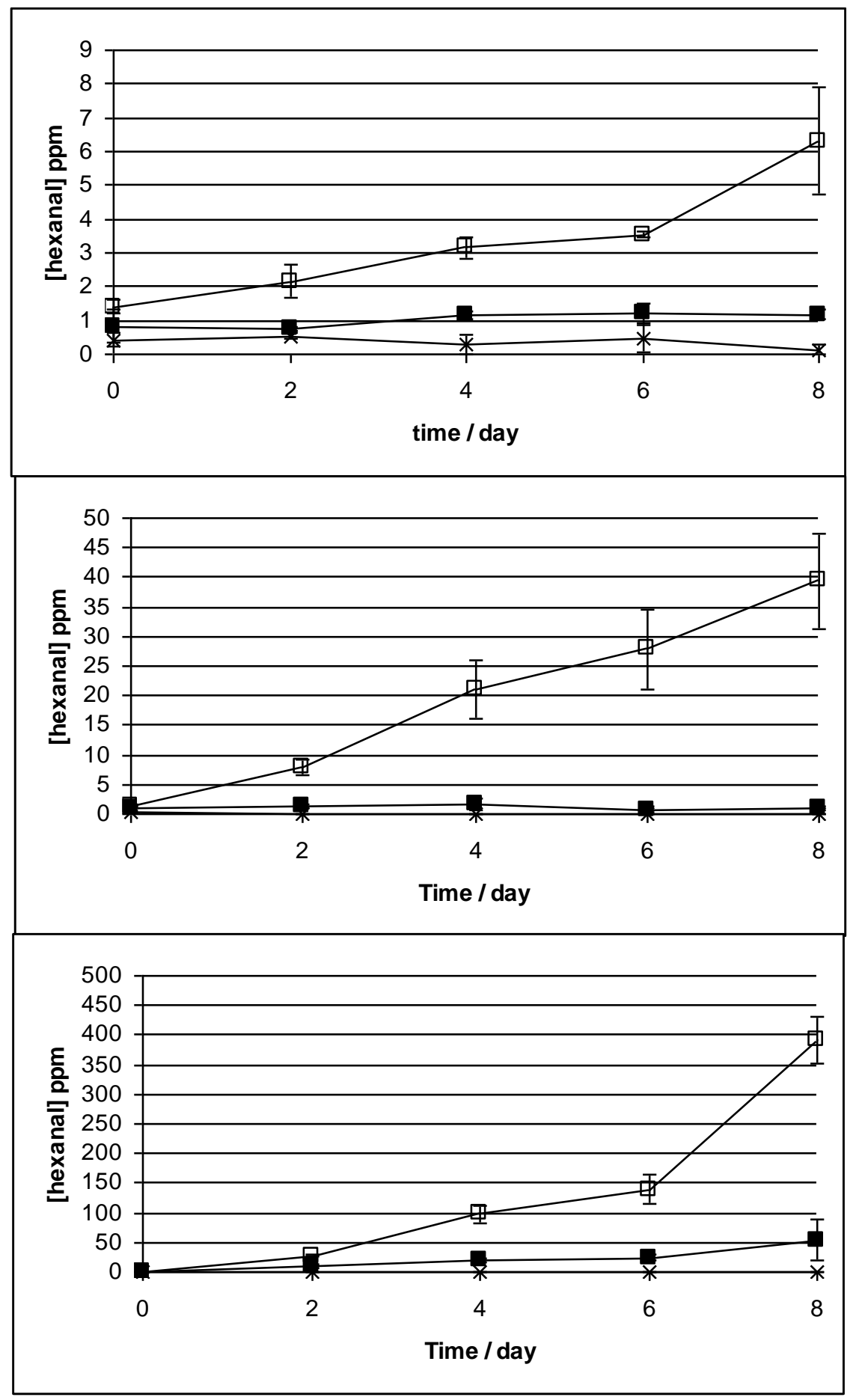

Figure 5: Influence of temperature on hexanal production in 10\% lipid emulsions stabilised by SDS (filled square), Tween 20 (cross) and DTAB (empty square). Extraction by solid phase microextraction and quantification by GC-MS. Emulsions were analysed at (a) $5^{\circ} \mathrm{C}$ (b) $25^{\circ} \mathrm{C}$ and (c) $45^{\circ} \mathrm{C}$. All data points represent means $(\mathrm{n}=3) \pm$ Standard Deviation. 